\title{
A Novel Self-Powered Wireless Sensor Node Based on Energy Harvesting for Mechanical Vibration Monitoring
}

\author{
Xihai Zhang, Junlong Fang, Fanfeng Meng, and Xiaoli Wei \\ School of Electrical and Information Engineering, Northeast Agricultural University, Harbin 150030, China \\ Correspondence should be addressed to Xihai Zhang; xhzhang@neau.edu.cn
}

Received 30 January 2014; Accepted 12 February 2014; Published 30 March 2014

Academic Editor: Weichao Sun

Copyright ( 2014 Xihai Zhang et al. This is an open access article distributed under the Creative Commons Attribution License, which permits unrestricted use, distribution, and reproduction in any medium, provided the original work is properly cited.

\begin{abstract}
Wireless sensor networks (WSNs) have been expected to improve the capability of capturing mechanical vibration dynamic behaviors and evaluating the current health status of equipment. While the expectation for mechanical vibration monitoring using WSNs has been high, one of the key limitations is the limited lifetime of batteries for sensor node. The energy harvesting technologies have been recently proposed. One of them shares the same main idea, that is, energy harvesting from ambient vibration can be converted into electric power. Employing the vibration energy harvesting, a novel self-powered wireless sensor node has been developed to measure mechanical vibration in this paper. The overall architecture of node is proposed. The wireless sensor node is described into four main components: the energy harvesting unit, the microprocessor unit, the radio transceiver unit, and accelerometer. Moreover, the software used to control the operation of wireless node is also suggested. At last, in order to achieve continuous self-powered for nodes, two operation modes including the charging mode and discharging mode are proposed. This design can effectively solve the problem of continuous supply power of sensor node for mechanical vibration monitoring.
\end{abstract}

\section{Introduction}

Mechanical vibration is a common phenomenon observed in the operation of many machines and arises from the inertia effect of machine parts in motion. Generally, the primary motivation for monitoring vibration in mechanical systems is to avoid causing component faults and early replacement and inflicting a major hit on accuracy. Therefore, efficient mechanical vibration monitoring is critical to machinery normal running. As we known, vibration monitoring system may be more complex than the other monitoring systems. A lot of signal cables in traditional vibration monitoring system are utilized, which results in signals interference and poor reliability of monitoring results. Moreover, the vibration monitoring system is also a limited application because the system is complex in construct and has poor flexibility $[1,2]$.

In order to overcome the shortcomings of traditional vibration monitoring system and achieve the vibration monitoring of machine within a large area, a method applying WSNs technology to vibration monitoring system of machine is presented. However, the power source applied for WSNs node still use batteries until now. The limited lifetimes of batteries have severely limited the potential application of WSNs for real engineering.

We know that utilizing ambient energy provides a new way that could potentially solve energy supply problem for real engineering. For example, mechanical vibration, existing almost everywhere, has been investigated as a promising energy source for wireless sensors in some applications, such as machine condition monitoring and indoor environmental monitoring [3-5].

Over the course of the past years, there has been a large amount of researches have been reported on energy harvesting [6-13]. They share the same main idea that energy harvesting from ambient vibration can be converted into electric power. At present, the most mature available method is based on solar cells. While solar cells are attractive for some outdoor applications, they are not useful for sensor nodes that are not accessible to the direct sunlight. However, these energy harvesting devices pick up vibrations from a vibrating source, and then convert vibration energy to electrical energy using piezoelectric materials. Therefore, energy harvesting 
from environmental vibration attracts much attention for its use in WSNs node [14]. Roundy et al. [15] research on the low level vibrations as a power source for wireless sensor nodes. Mitcheson et al. [16] review energy harvesting from human and machine motion for wireless electronic devices. Vullers et al. [17] summarize energy harvesting components and associated power management circuits. Such system can be used in various applications such as mechanical vibration monitoring systems and building energy management systems, et al. Hence, energy harvesting technology has become powerful engineering approach for solving energy problem of wireless sensor node. But to the best of the author's knowledge, it seems that the self-powered wireless sensor node utilized vibration energy harvesting for mechanical vibration monitoring has been seldom addressed, which motivates our research in this paper.

Following the above discussions on related content, we present firstly the overall system architecture of sensor node. The wireless sensor node is described into four main components: the energy harvesting unit, the microprocessor unit, the radio transceiver unit, and accelerometer unit. Each subsystem will be described in detail in their respective sections. Moreover, the software design of wireless sensor node includes ZigBee stack and wireless sensor communication. At last, in order to achieve continuous self-powered, two operation modes of energy storage module including the charging mode and discharging mode are suggested in this paper.

The rest of paper is organized as follows. The energy harvesting is introduced in Section 2. The hardware system architecture is given in Section 3. Then, the software design of sensor node is proposed in Section 4. At last, the two kinds of supply power mode are analyzed in Section 5 and the paper is concluded in Section 6.

\section{Energy Harvesting}

Over the past several years, energy harvesting has been improved rapidly to deliver devices that can provide continual power output from vibrations on a vibrating source using piezoelectric materials, electromagnetic induction, or electrostatic methods. Therefore, main idea of this paper is that wireless sensor node is powered by the vibration energy harvesting which can convert the wasted vibration to useful electrical energy.

Here, a new piezoelectric energy harvester is proposed for mechanical vibration energy harvesting. Piezoelectric energy harvesters have high output voltage but low current level. They have simple structures, which makes them compatible with Micro-Electro-Mechanical Systems (MEMS). Linear Technology's new LTC3588-1 piezoelectric energy harvesting power supply greatly simplifies the task of harvesting surplus energy and managing the piezoelectric energy from a vibration or strain source.

Linear Technology announces the LTC3588-1, a complete energy harvesting solution optimized for low energy sources, including piezoelectric transducers. The LTC3588-1 integrates a low-loss, full-wave bridge rectifier with a high efficiency buck converter to harvest ambient vibrational energy

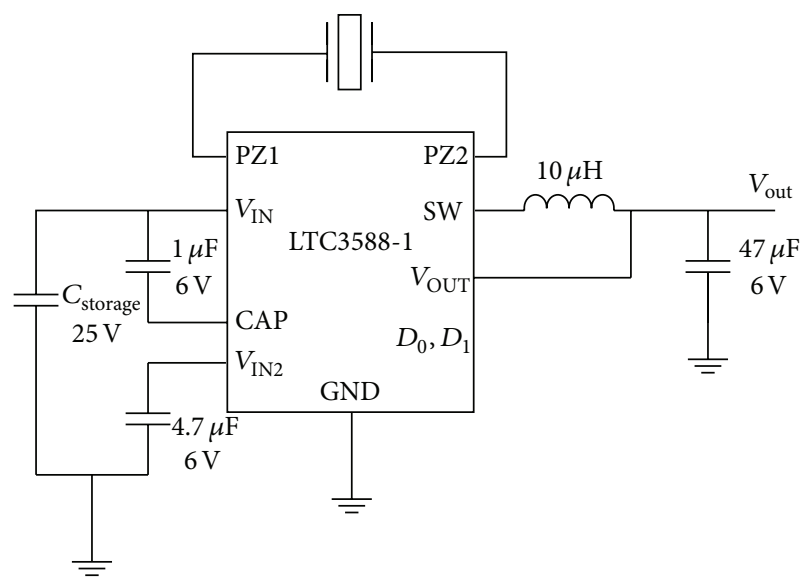

FIGURE 1: LTC3588 typical application schematic.

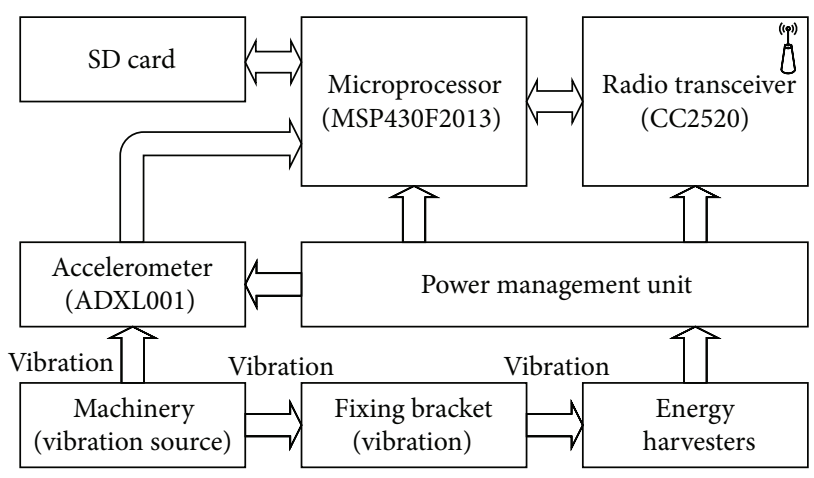

FIGURE 2: Block diagram of wireless sensor node.

via piezoelectric transducers and then convert it to a wellregulated output to power. The LTC3588-1 operates from an input voltage range of $2.7 \mathrm{~V}$ to $20 \mathrm{~V}$, making it ideal for a wide array of piezoelectric transducers, as well other high output impedance energy sources. Its high efficiency buck DC/DC converter delivers up to $100 \mathrm{~mA}$ of continuous output current or even higher pulsed loads. Its output can be programmed to one of four $(1.8 \mathrm{~V}, 2.5 \mathrm{~V}, 3.3 \mathrm{~V}$, or $3.6 \mathrm{~V})$ fixed voltages to power a wireless transmitter or sensor. Quiescent current is only $950 \mathrm{nA}$ with the output in regulation, maximizing overall efficiency [18].

Linear's Technology LTC3588-1 chip integrates all necessary blocks for implementation of piezoelectric energy harvesting devices and the typical application of $100 \mathrm{~mA}$ piezoelectric energy harvesting power supply is shown in Figure 1.

\section{System Architecture}

The wireless sensor node is composed of several parts: the microprocessor unit, the radio transceiver unit, the accelerometer unit, and energy harvesting unit. The overall system hardware block diagram is shown in Figure 2. Microprocessor, which uses microprocessor MSP430F2013, 
is mainly responsible for controlling the operation of the entire data acquisition node processing. Sensor unit is used to collect machinery and equipment vibration parameters. In this system, the accelerometer uses vibration sensor (ADXL001) which is specially for equipment status monitoring. RF CC2520 chip is as the radio transceiver unit which is mainly responsible for data transmission communication with other sensor nodes, sending and receiving data. The energy harvesting unit is in charge of providing a stable and reliable power supply voltage to sensor node and ensuring that nodes are able to work properly. This unit is described in detail in Section 2. Another subsystem will be illustrated in their respective subsections.

3.1. Microprocessor Unit. The microprocessor is the heart of sensor node. It samples the sensor data and handles the radio communication. Because the energy harvesting unit harvests energy at a relatively low rate, it is important to select a microprocessor with low power consumption. The MSP430 family has the best performance in terms of power consumption and has a large community with support for many low power applications. The architecture, combined with five low-power modes, is optimized to achieve extended battery life in portable measurement applications. The device features a powerful 16-bit RISC CPU, 16-bit registers, and constant generators that contribute to maximum code efficiency. Therefore, in this system, microprocessor MSP430 is selected.

The MSP430F2013 is an ultra-low-power mixed signal microprocessor with a built-in 16-bit timer and ten I/O pins. In addition, there is already an 802.15.4 library written for the MSP430F2013 by TI and also the CC2520 transceiver datasheet provides routing details between the microprocessor and the CC2520 transceiver.

3.2. Radio Transceiver Unit. In this design, the CC2520 wireless module is selected as radio transceiver unit. The CC2520 wireless module uses ZigBee/IEEE 802.15.4 RF transceiver for the $2.4 \mathrm{GHz}$ unlicensed ISM band. In this self-powered sensor node, this module can be used together with a microprocessor MSP430F2013.

The CC2520 is designed for low-power consumption at $18.5 \mathrm{~mA}$ when receiving and $25.8 \mathrm{~mA}$ when transmitting. The microprocessor is designed with a hardware SPI interface. The CC2520 interfaced with the microprocessor via the 4wire SPI bus: CSn, SI, SO, and SCLK. The SPI interface provided synchronous full duplex communication consisting of three lines which are active low. The CSn pin is a digital input for SPI chip select. The SI pin is also a digital input for SPI slave input. The next input pin on the serial peripheral interface of the wireless controller is SCLK. This pin is an SPI clock input. The final line Serial Out (SO) is used to transmit data from the transceiver to the master device which was the microprocessor. Moreover, it enables ZigBee nodes to be built with very low total bill-of material costs. Therefore, the CC2520 is highly suited for systems where a high data sampling rate with low energy consumption is required in mechanical vibration monitoring.

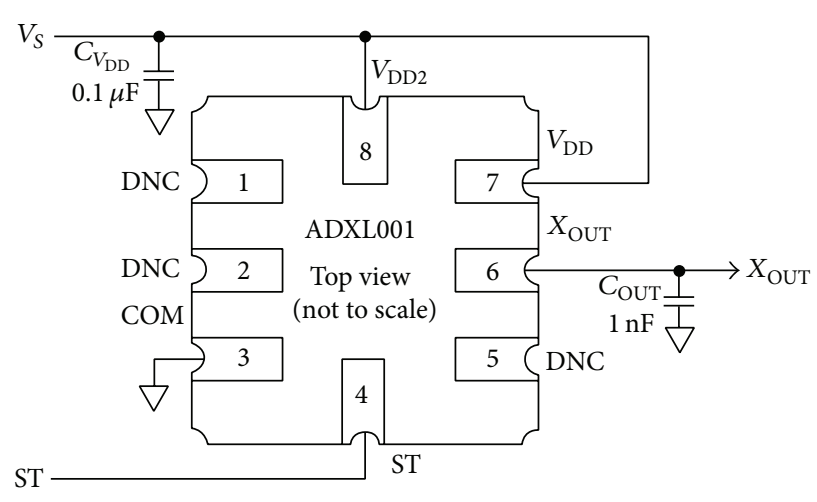

$\mathrm{DNC}=$ do not connect

FIgUre 3: Application circuit.

3.3. Accelerometer. It is determined that the frequency range of interest for this self-powered sensor node application is defined between $10 \mathrm{~Hz}$ and $20 \mathrm{kHz}$. The best fit for application is the ADXL001, an MEMS based accelerometer developed by Analog Devices which has a bandwidth of $22 \mathrm{kHz}$, operates at $5 \mathrm{~V}$, and outputs an analog voltage.

The ADXL001 provides high performance and wide bandwidth for industrial monitoring where wide bandwidth, small form factor, low power, and high performance are critical. Unlike many other vibration sensors, which operate below $5 \mathrm{kHz}$ of bandwidth, the ADXL001 is capable of detecting motor-bearing vibration and irregularities up to $22 \mathrm{kHz}$, which allows system operators to identify failing equipment earlier before costly damage is sustained. Moreover, the sensors have excellent nonlinearity of 0.2 percent of full-scale range. The ADXL001 operates with an extended temperature range of $-40^{\circ} \mathrm{C}$ to $125^{\circ} \mathrm{C}$ allowing the part to be used in most hazardous industrial conditions. And the ADXL001 functions on a $3.3 \mathrm{~V}$ to $5 \mathrm{~V}$ supply.

Figure 3 shows the standard application circuit for the ADXL001. Figure 4 is appearance of ADXL001 chip. Note that $V_{\mathrm{DD}}$ and $V_{\mathrm{DD} 2}$ should always be connected together. The output is shown connected to a $1000 \mathrm{pF}$ output capacitor for improved electromagnetic interference (EMI) performance and can be connected directly to an ADC input. Use standard best practices for interfacing with an ADC and do not omit an appropriate antialiasing filter [19].

\section{Design of Node Software}

The software used to control the operation of the node has been written in $\mathrm{C}$ and then cross-compiled using the IAR Embedded Workbench development environment for the CC2520. Function libraries supplied for use with the development environment enable the control and operation of the various peripherals within CC2520.

Two programs are defined in CC2520 wireless module. One is responsible for sending and receiving radio messages, that is, receiving a command message from the node and 


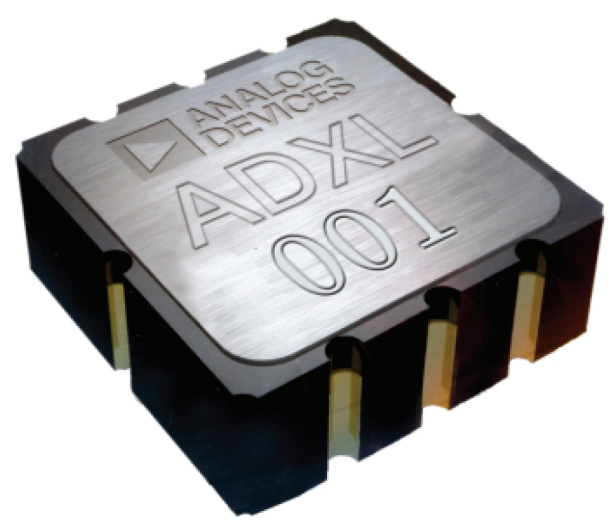

Figure 4: ADXL001.

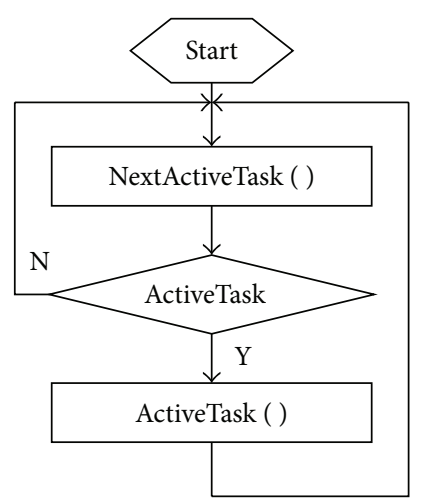

FIgURE 5: OSAL task scheduling mechanism. sending message to the monitoring terminal. Another program is responsible for MSP430F2013 chip communication, that is, sending control commands through the serial port and receiving the collected data from the serial port. Two application program objects interact mutually by a message mechanism of OSAL and collaborate together to complete the program functions on the node. The OSAL task scheduling is shown in Figure 5. NextActiveTask( ) is task event query function what returns the ActiveTask. When we design software, we can decide if ActiveTask ( ) is executed according to the value of ActiveTask.

The MSP430F2013 chip implements data acquisition and storage capabilities. The flow chart of mechanical vibration signal acquisition is shown in Figure 6. After system powerup, the initialization of hardware platform and each software module should be completed. As a result of dual processor in this node design, the initialization of system consists of two parts: MSP430F2013 and CC2520. After data collection begins, the vibration signal is sampled through ADXL001 and then the obtained data are sent and stored temporary into the Flash. When Flash is almost full, the interrupt request signal is sent to MSP430F2013. Then MSP430F2013 begin to read data and storage data into SD card. Data in SD card is sent to radio transceiver unit (CC2520) through USART. After the completion of transmission, the MSP430F2013 will wait for the next interrupt request and then continue to transmit data.

\section{Power Mode Analyses}

The work course of self-powered wireless sensor node is as follows. The piezoelectric power generating module generates a weak AC signal by the piezoelectric bending transducer. And then power generated is send into the energy storage module. This energy storage module has two modes of operation: the charging mode and the discharging mode. In charging mode, wireless sensor nodes are intermittent work and the energy harvester is first in charging mode under the continuous vibration environment. Power generated by the piezoelectric transducer is stored in the capacitor of the LTC3588-1 and is converted into the standard output voltage 3.3 $\mathrm{V}$ for the node. Meanwhile, the backup battery is charged by the power stored in the $C_{\text {storage }}$ of Figure 1 . In discharging mode, when the piezoelectric power generation module cannot output charge and the voltage of LTC3588-1's $V_{\text {IN }}$ decline, the rechargeable batteries begin to charge for $C_{\text {storage }}$ in Figure 1, so that the node is under the normal operation status. Therefore, if energy harvesting is under work, the charging mode and discharging mode run alternately.

\section{Conclusions}

This paper introduces a novel design of self-powered wireless sensor node based on energy harvesting for mechanical vibration monitoring. Firstly, the overall architecture of node is proposed. The wireless sensor node design is described into four main components: the energy harvesting unit (LTC35881 ), the microprocessor unit (MSP430F2013), and the radio transceiver unit (CC2520) and accelerometer (ADXL001). Moreover, the software used to control the operation of the node is also suggested. At last, in order to achieve continuous self-powered for nodes, two operation modes of energy storage module including the charging mode and the discharging mode are proposed. This design can effectively solve the problem of continuous power supply for sensor node. However, there are a number of open problems worth pursuing. One concerns that the energy harvesting should be designed according to the specific source of mechanical vibration, which requires a large amount of mechanical vibration prior accumulated data. The other concerns that the most reasonable duty cycle needs to be designed to acquire sufficient power from energy harvesting in the node sleep time so as to meet the requirements of a working node.

\section{Conflict of Interests}

The authors declare that there is no conflict of interests regarding the publication of this paper.

\section{Acknowledgments}

This research was supported by the National Natural Science Foundation of China (Grant No. 31101080). The authors 


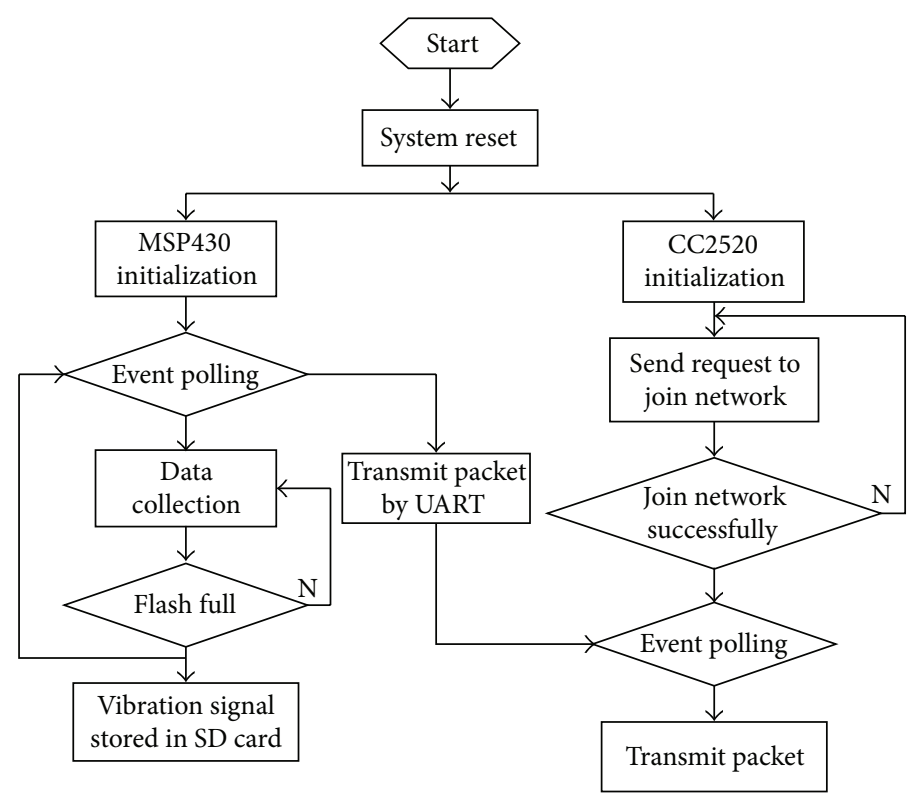

FIGURE 6: Vibration signal acquisition process.

would like to thank the anonymous reviewers for their helpful suggestions, which greatly improve the paper.

\section{References}

[1] J. Yick, B. Mukherjee, and D. Ghosal, "Wireless sensor network survey," Computer Networks, vol. 52, no. 12, pp. 2292-2330, 2008.

[2] A. Vogl, T. W. Dag, P. Storas et al., "Design, process and characterisation of a high-performance vibration sensor for wireless condition monitoring," Sensors and Actuators A: Physical, vol. 153, no. 2-3, pp. 155-161, 2009.

[3] S. Roundy and P. K. Wright, "A piezoelectric vibration based generator for wireless electronics," Smart Materials and Structures, vol. 13, no. 5, pp. 1131-1142, 2004.

[4] E. P. James, M. J. Tubor, S. P. Beeby et al., "An investigation of self-powered systems for condition monitoring applications," Sensors and Actuators A: Physical, vol. 110, no. 1-3, pp. 171-176, 2004.

[5] E. S. Leland, E. M. Lai, and P. K. Wright, "A self-powered wireless sensor for indoor environmental monitoring," in Proceedings of the Wireless Networking Symposium, Austin, Tex, USA, October 2004.

[6] S. Roundy, P. K. Wright, and K. S. J. Pister, "Micro-electrostatic vibration-to-electricity converters," in Proceedings of the ASME International Mechanical Engineering Congress and Exposition (IMECE '02), pp. 487-496, New Orleans, La, USA, November 2002.

[7] S. Roundy, P. K. Wright, and J. Rabaey, "A study of low level vibrations as a power source for wireless sensor nodes," Computer Communications, vol. 26, no. 11, pp. 1131-1144, 2003.

[8] P. Glynne-Jones, M. J. Tudor, S. P. Beeby, and N. M. White, “An electromagnetic vibration-powered generator for intelligent sensor systems," Sensors and Actuators A: Physical, vol. 110, no. 1-3, pp. 344-349, 2004.

[9] P. D. Mitcheson, T. C. Green, E. M. Yeatman, and A. S. Holmes, "Architectures for vibration-driven micropower generators,"
Journal of Microelectromechanical Systems, vol. 13, no. 3, pp. 429-440, 2004.

[10] G. Poulin, E. Sarraute, and F. Costa, "Generation of electrical energy for portable devices: comparative study of an electromagnetic and a piezoelectric system," Sensors and Actuators A: Physical, vol. 116, no. 3, pp. 461-471, 2004.

[11] D. Guyomar, A. Badel, E. Lefeuvre, and C. Richard, “Toward energy harvesting using active materials and conversion improvement by nonlinear processing," IEEE Transactions on Ultrasonics, Ferroelectrics, and Frequency Control, vol. 52, no. 4, pp. 584-595, 2005.

[12] C. R. Saha, T. O’Donnell, H. Loder, S. Beeby, and J. Tudor, "Optimization of an electromagnetic energy harvesting device," IEEE Transactions on Magnetics, vol. 42, no. 10, pp. 3509-3511, 2006.

[13] Y. C. Shu and I. C. Lien, "Analysis of power output for piezoelectric energy harvesting systems," Smart Materials and Structures, vol. 15, no. 6, article 001, pp. 1499-1512, 2006.

[14] K. Matsumoto, K. Saruwatari, and Y. Suzuki, "Vibrationpowered battery-less sensor node using electret generator," in Proceedings of the 11th International Workshop on Micro and Nanotechnology for Power Generation and Energy Conversion Applications, pp. 134-137, Seoul, Republic of Korea, November 2011.

[15] S. Roundy, P. K. Wright, and J. Rabaey, "A study of low level vibrations as a power source for wireless sensor nodes," Computer Communications, vol. 26, no. 11, pp. 1131-1144, 2003.

[16] P. D. Mitcheson, E. M. Yeatman, G. K. Rao, A. S. Holmes, and T. C. Green, "Energy harvesting from human and machine motion for wireless electronic devices," Proceedings of the IEEE, vol. 96, no. 9, pp. 1457-1486, 2008.

[17] R. J. M. Vullers, R. van Schaijk, I. Doms, C. van Hoof, and R. Mertens, "Micropower energy harvesting," Solid-State Electronics, vol. 53, no. 7, pp. 684-693, 2009.

[18] http://www.linear.com/product/LTC3588-1.

[19] http://www.analog.com/static/imported-files/data_sheets/ ADXL001.pdf. 


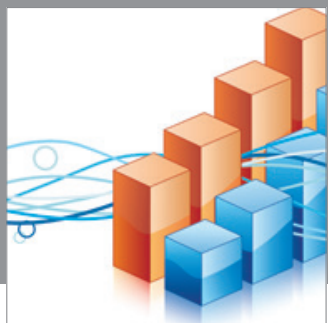

Advances in

Operations Research

mansans

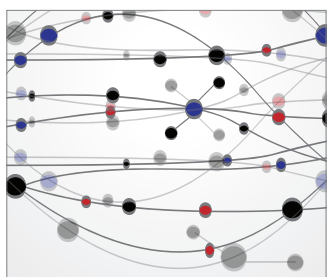

The Scientific World Journal
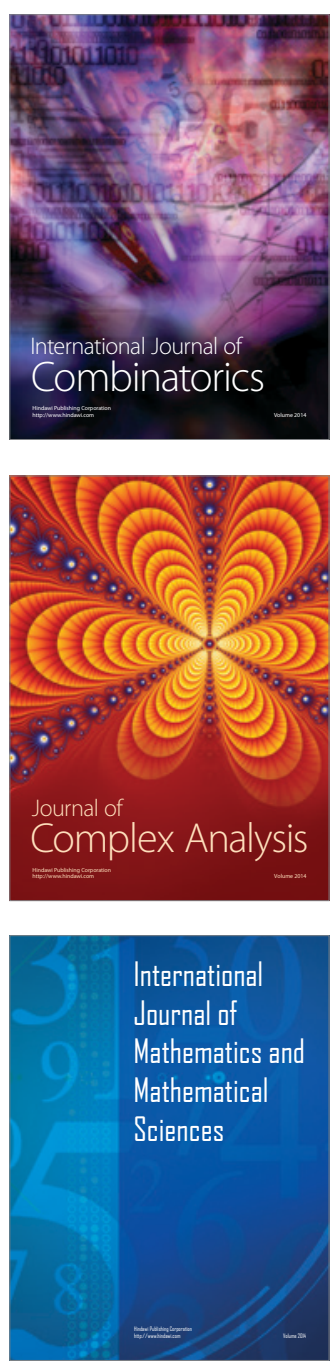
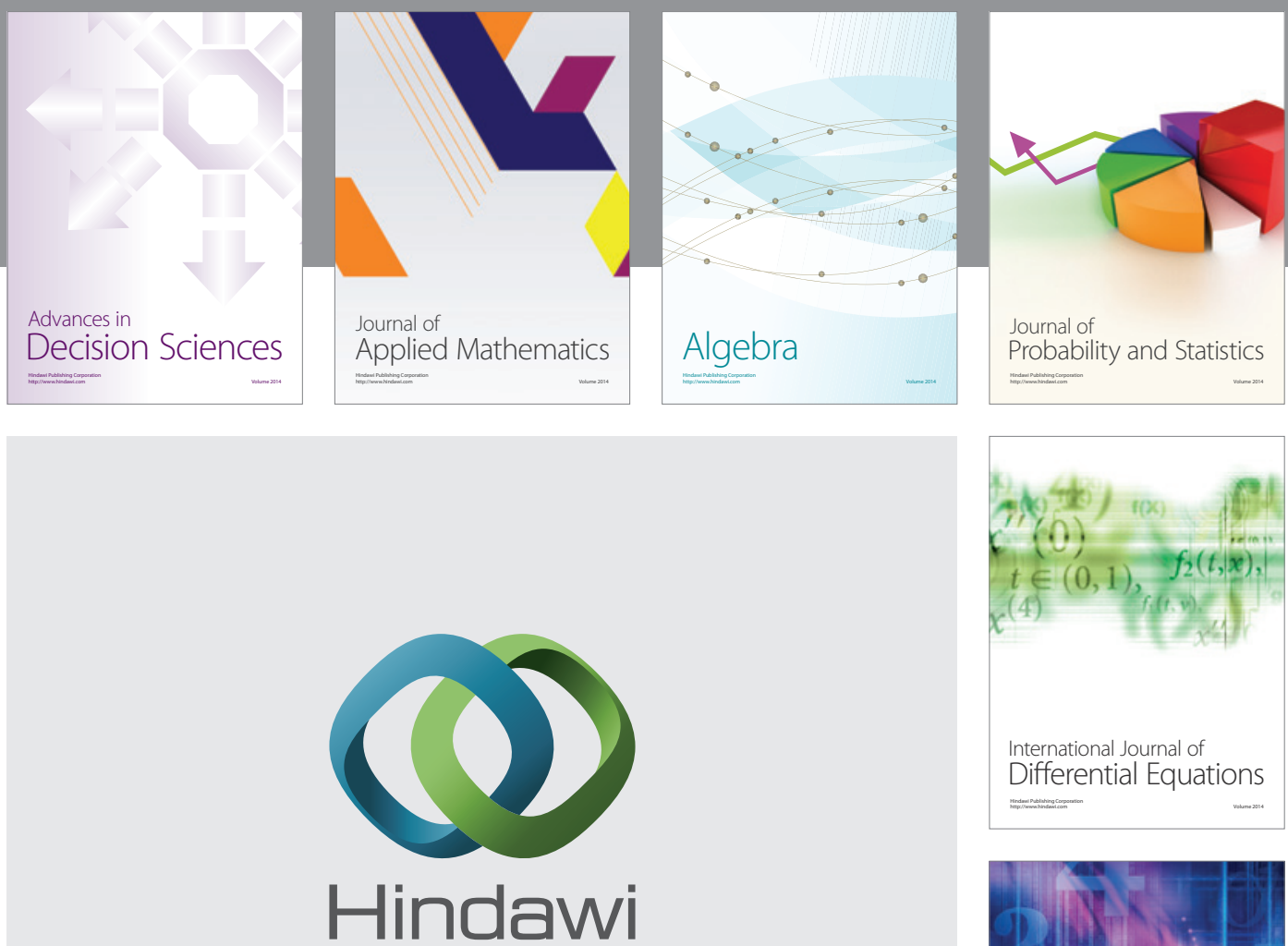

Submit your manuscripts at http://www.hindawi.com
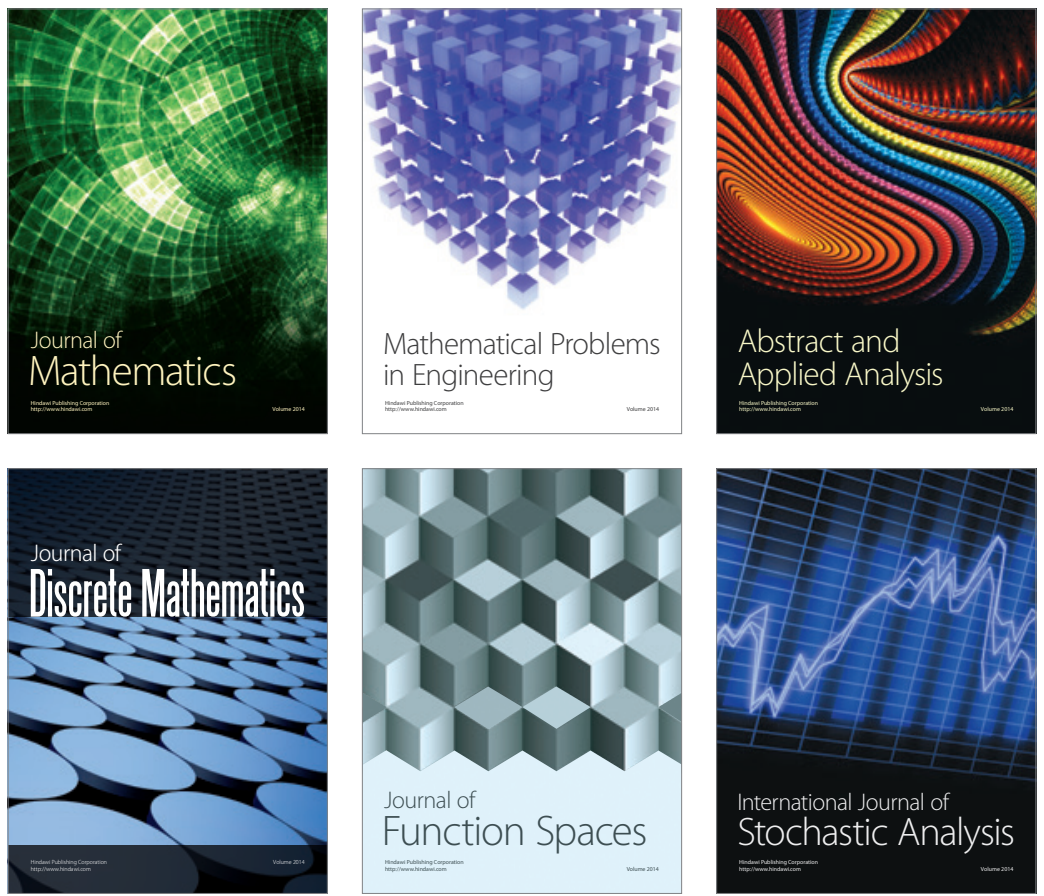

Journal of

Function Spaces

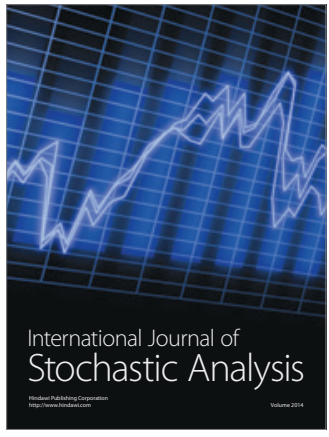

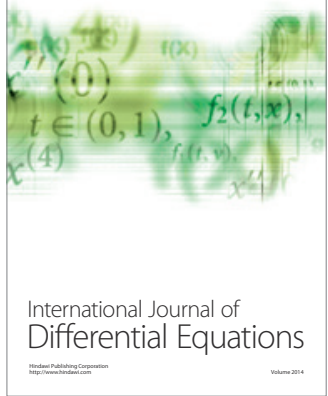
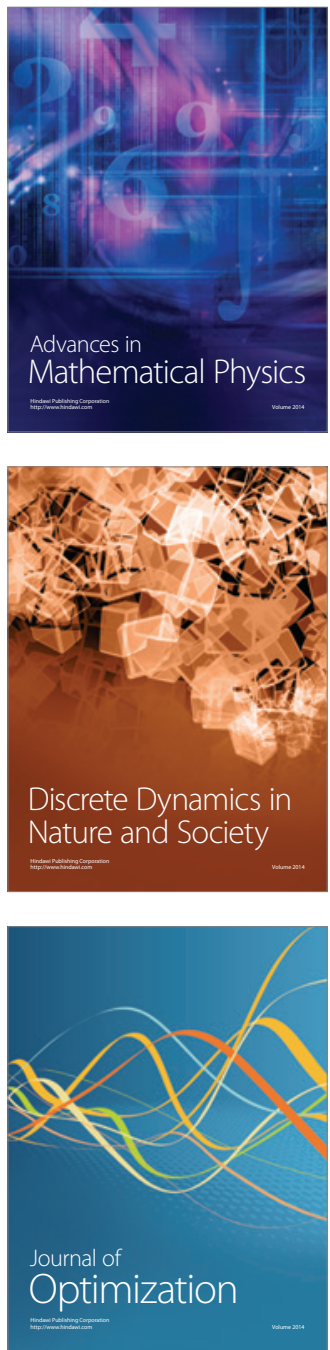